\title{
Characterization of chronic arthritis in a multicenter study of 852 childhood-onset systemic lupus erythematosus patients
}

\author{
Ana Paula Sakamoto ${ }^{1}$ Clovis Artur Silva ${ }^{2,3} \cdot$ Mariana Paes Leme Ferriani $^{2} \cdot$ Rosa Maria Rodrigues Pereira $^{3} \cdot$ \\ Eloisa Bonfá ${ }^{3}$ - Claudia Saad-Magalhães ${ }^{4}$ Eunice Okuda $^{5} \cdot$ Simone Appenzeller $^{6} \cdot$ Francisco Hugo Gomes $^{7}$. \\ Ana Luiza Garcia Cunha ${ }^{1} \cdot$ Mirna Henriques Tomich Salume ${ }^{1} \cdot$ Daniela Petry Piotto $^{1} \cdot$ Maria Teresa Terreri $^{1}$
}

Received: 23 May 2016 / Accepted: 1 September 2016 / Published online: 14 September 2016

(C) Springer-Verlag Berlin Heidelberg 2016

\begin{abstract}
Chronic arthritis (CA) is an unusual condition in childhood-onset systemic lupus erythematosus (cSLE) and data in children is very limited. The aim of the study is to assess CA in a large population of cSLE patients, in a multicenter cross-sectional study including 852 cSLE patients followed in ten Pediatric Rheumatology referral services in state of São Paulo, Brazil. CA was observed in 32/852 (3.7 \%) cSLE patients mostly in hands and ankles. Chronic monoarthritis was diagnosed in four cSLE patients, oligoarthritis in nine and polyarthritis in 19. In the latter group, six had rhupus syndrome. Two oligoarticular patients had Jaccoud's arthropathy. CA was an isolated manifestation observed at disease onset in 13/32 (41\%) cSLE patients, and juvenile idiopathic arthritis (JIA) was the first diagnosis in 18/32 (56\%). The comparison of last visit of patients
\end{abstract}

Maria Teresa Terreri

teterreri@terra.com.br

1 Pediatric Rheumatology Unit, Universidade Federal de São Paulo, Rua Borges Lagoa 802, São Paulo, SP 04083-001, Brazil

2 Pediatric Rheumatology Unit, Faculdade de Medicina da Universidade de São Paulo (USP), São Paulo, Brazil

3 Division of Rheumatology, Faculdade de Medicina da Universidade de São Paulo (USP), São Paulo, Brazil

4 Pediatric Rheumatology Division, São Paulo State University (UNESP) - Faculdade de Medicina de Botucatu, São Paulo, Brazil

5 Pediatric Rheumatology Unit, Irmandade da Santa Casa de Misericórdia de São Paulo, São Paulo, Brazil

6 Pediatric Rheumatology Unit, State University of Campinas (UNICAMP), São Paulo, Brazil

7 Pediatric Rheumatology Unit, Ribeirão Preto Medical School, University of São Paulo, São Paulo, Brazil with CA and without this manifestation revealed higher frequency of splenomegaly (28 vs. $11 \%, p=0.002$ ). The median of SLICC/ACR-DI score $[1(0-9)$ vs. $0(0-7)$, $p=0.003]$ was significantly higher in CA patients compared to patients without this manifestation, likewise the frequency of musculoskeletal damage ( 31 vs. $9 \% p=0.001$ ). Frequencies of treatment with nonsteroidal anti-inflammatory drugs ( 75 vs. $26 \%, p<0.0001)$, hydroxychloroquine sulfate (87 vs. $59 \%, p=0.001$ ) and methotrexate (47 vs. $22 \%, p=0.001)$ were significantly higher in CA patients. This large multicenter study allowed us to characterize CA as a rare and early manifestation of cSLE, frequently mimicking JIA at disease onset. It is predominantly polyarticular, involving more often hands and ankles and it is associated with significant musculoskeletal accrual damage.

Keywords Childhood - Chronic arthritis · Rhupus · Jaccoud's arthropathy · Childhood-onset systemic lupus erythematosus $\cdot$ Systemic lupus erythematosus

\section{Introduction}

Systemic lupus erythematosus (SLE) is a rare autoimmune disease more common in adults (aSLE) with only 10-20\% of cases beginning during childhood or adolescence [1]. Childhood-onset SLE (cSLE) is characterized by the involvement of various organs and systems with different disease presentations and leading to significant morbidity and mortality [2].

Acute articular involvement is one of the most common and earliest symptoms at cSLE onset. It has been reported in 60-90 \% of cSLE patients and it is typically symmetric, nonerosive and nondeforming, with polyarticular involvement, affecting both large and small joints [2,3]. 
On the other hand, chronic arthritis (CA) is an unusual condition in aSLE $[4,8]$ and in cSLE [3, 6]. Data in children has been limited mostly to case reports $[3,5-7]$ and to a large single center study comparing adults and children with CA focusing solely on distinct features in these two age brackets [8]. There are, however, no data regarding the possible clinical associations of CA in cSLE patients with and without this condition.

Therefore, the objective of this study was to assess CA in a large tertiary multicenter population of cSLE patients to evaluate the possible demographic and cumulative clinical, laboratorial and treatment associations with this musculoskeletal manifestation.

\section{Methods}

\section{Study design and patients}

A multicenter cross-sectional study was performed including all the consecutive cSLE patients followed in ten Pediatric Rheumatology referral services in the state of São Paulo, Brazil. Exclusion criteria were: incomplete medical charts, undifferentiated connective tissue disorder with three or fewer American College of Rheumatology (ACR) criteria for SLE [9], isolated cutaneous lupus erythematosus, neonatal lupus erythematosus, drug-induced lupus and other autoimmune diseases. The remaining cSLE patients fulfilled the ACR criteria for SLE [9], with disease onset before 18 years of age [10] and current age up to 25 years old. The cohort was evaluated at the time of the diagnosis and at patients' last visit. In all the participant centers, the Committee for Research Ethics approved the study.

An investigator meeting was held for this study to define the protocol, including definitions of clinical, laboratory and treatment parameters and disease activity and damage score. All investigators used the same specific database.

All patient's medical charts were reviewed according to an extensive standardized protocol for demographic data, CA characteristics, other clinical features, laboratorial findings, therapeutic data and outcome (articular deformity, cumulative damage and death). CA was defined as the presence of swelling or effusion or two or more of the following: limitation in the range of joint movement, pain or tenderness on motion and increased temperature for at least 6 weeks $[8,11]$. Arthritis features were also evaluated: duration, number and type of joints with arthritis, number and type of joints with limitation in range of motion and deformity, and radiographic changes (juxta-articular osteopenia, joint-space narrowing and erosion). Rhupus was characterized as the association of cSLE and chronic inflammatory arthritis with erosive damage with or without positive rheumatoid factor $[3,5,6,8]$. Jaccoud's arthropathy was diagnosed as a nonerosive arthropathy with marked articular dislocation or subluxation leading to severe deformity of the hands and feet. [8, 12].

\section{Demographic data, clinical evaluation, disease activity, disease damage and drug therapy}

Demographic data analysis included: age at cSLE onset, disease duration, age at last visit, gender and ethnicity. Descriptors and definitions of disease activity regarding SLE clinical manifestations were based on SLE Disease Activity Index 2000 (SLEDAI-2 K) score, and were evaluated at last visit [13]. Other cumulative SLE clinical manifestations included fever (axillary temperature higher than $37.8^{\circ} \mathrm{C}$ ), weight loss $>2 \mathrm{~kg}$, lymphadenopathy (peripheral lymph node enlargement $>1.0 \mathrm{~cm}$ ), hepatomegaly [based on physical exam with liver edge $\geq 2 \mathrm{~cm}$ below the right costal margin or imaging (ultrasound or computer tomography when available)] and splenomegaly [based on physical exam with palpable spleen or imaging (ultrasound or computer tomography when available)] [14]. Neuropsychiatric lupus included 19 syndromes according to ACR classification criteria [15]. Antiphospholipid syndrome was diagnosed according to the preliminary criteria for the classification of pediatric antiphospholipid syndrome [16]. High blood pressure was defined as systolic and/or diastolic blood pressures $\geq 95$ th percentile for gender, age and height on $\geq 3$ occasions [17]. Acute kidney injury was determined by sudden increase in serum creatinine above $2 \mathrm{mg} / \mathrm{dl}$ [18] or by modified RIFLE (Risk, Injury, Failure, Loss of kidney function and End-stage kidney disease) criteria [19]. Chronic renal disease was defined as structural or functional abnormalities of the kidney for $\geq 3$ months (with or without decreased glomerular filtration rate) or glomerular filtration rate $<60 \mathrm{ml} / \mathrm{min} / 1.73 \mathrm{~m}^{2}$ for $\geq 3$ months [20].

Laboratorial assessment included retrospective analysis of erythrocyte sedimentation rate (ESR), C-reactive protein (CRP), complete blood cell count, serum urea and creatinine, urinalysis and 24-hour urine protein excretion. Complement levels (CH50, C3 and C4) were assessed by immunodiffusion, turbidimetric immunoassay or immunonephelometry. Antinuclear antibodies (ANA) were tested by indirect immunofluorescence; anti-double-stranded DNA (anti-dsDNA) by indirect immunofluorescence or enzyme linked immuno sorbent assay (ELISA); anti-Sm and anti-RNP by passive hemagglutination or ELISA; antiSSA/Ro and anti-SSB/La by contraimmunoelectrophoresis or ELISA; anticardiolipin (aCL) IgG and IgM autoantibodies by ELISA. All the autoantibodies profiles were carried 
out at each center. The cutoff values from the kit manufacturer were used to define abnormal. Lupus anticoagulant was detected according to the guidelines of the International Society on Thrombosis and Hemostasis [21]. Further characterization of CA patients included rheumatoid factor determination by latex fixation test, nephelometry or ELISA and conventional radiography of the affected joints.

SLE disease activity and cumulative damage were scored through the SLEDAI-2K [13] and the Systemic Lupus International Collaborating Clinics/ACR-Damage Index (SLICC/ACR-DI) [22], respectively.

Cumulative drug treatment data (prednisone, intravenous methylprednisolone, chloroquine diphosphate, hydroxychloroquine sulfate, methotrexate, azathioprine, cyclosporine, mycophenolate mofetil, intravenous cyclophosphamide, intravenous immunoglobulin, rituximab and plasmapheresis) were also recorded.

Patients were divided in two groups for the assessment of cSLE cumulative clinical manifestations, cumulative laboratory tests and autoantibodies evaluation and cumulative treatment at last visit: patients with $\mathrm{CA}$ and without CA.

Results were presented as numbers (\%) for categorical variables and median (range) or mean $\pm \mathrm{SD}$ for continuous variables. Categorical variables comparisons were assessed by Pearson $\chi$-Square or Fisher's exact test and continuous variables comparisons by Mann-Whitney test or $t$ test. The significance levels of the independent variable were set at $5 \%(p<0.05)$. Holm-Bonferroni correction for multiple comparisons was performed adjusting the significance level $(p<0.0038)$.

\section{Results}

The study was performed including 1017 cSLE patients followed in the participant's referral services. One hundred and sixty-five patients were excluded due to: incomplete medical charts $(n=96)$, undifferentiated connective tissue disorder with three or fewer ACR criteria $(n=43)$, isolated cutaneous lupus erythematosus $(n=11)$, neonatal lupus erythematosus $(n=8)$, drug-induced lupus $(n=5)$ and other autoimmune diseases $(n=2)$. All the remaining 852 cSLE patients fulfilled the inclusion criteria.

CA was found in 32/852 (3.7 \%) cSLE patients. CA is usually an early disease manifestation with a median period between arthritis and cSLE diagnosis of 1.0 month. The arthritis lasted up to 70 months with a median duration of 4.5 months. In 13/32 (41\%) patients CA occurred as an isolated manifestation before lupus diagnosis. Juvenile idiopathic atrthritis (JIA) was the first diagnosis in 18/32 (56\%) patients. The most frequently involved joints were small joints of the hands such as proximal interphalangeal joints $(25 \%)$ and metacarpophalangeal joints $(18 \%)$ followed by ankles $(16 \%)$, knees $(12 \%)$ and wrists $(11 \%)$. Chronic monoarthritis was observed in four $(13 \%)$ cSLE patients, oligoarthritis in nine $(28 \%)$ and polyarthritis in $19(59 \%)$ patients. In the latter group, 6/19 (31\%) had rhupus syndrome (all of them with joint erosion and three with positive rheumatoid factor). Among the oligoarthritis group 2/9 (22\%) had concomitantly CA of knees and Jaccoud's arthropathy (involving flexion contracture and ulnar deviation of the metacarpophalangeal joints). Deformity and radiographic changes were observed in about one third of patients. Joints with limitation of range of motion were more frequent in proximal interphalangeal joint (21\%), metacarpophalangeal joint (19\%), wrists (13\%), ankles $(10 \%)$ and elbows $(10 \%)$. The features of chronic arthritis in 32/852 cSLE are shown in Table 1.

Further comparison of demographic data and cumulative clinical manifestations in 852 cSLE patients with and without $\mathrm{CA}$ at the last visit are illustrated in Table 2. After Holm-Bonferroni correction for multiple comparisons $(p<0.0038)$, the only statistically significant differences were the time of follow-up since the diagnosis [5.7 (0.7$14.8)$ vs. $4.5(0-23.2)$ years, $p<0.0001]$ and frequency of splenomegaly ( 28 vs. $11 \%, p=0.002$ ) that were both higher in cSLE patients with CA compared to those without this manifestation (Table 2). The frequencies of other cumulative clinical manifestations were similar in both groups ( $p>0.0038$, Table 2$)$.

Disease activity and cumulative damage as well as laboratory tests and autoantibodies evaluation of 852 cSLE patients are shown in Table 3. After Holm-Bonferroni correction for multiple comparisons $(p<0.0038)$, the median of SLICC/ACR-DI score [1 (0-9) vs. $0(0-7), p=0.003$ ] was significantly higher in CA patients compared to patients without this complication. Further analysis of organ damage by system revealed that frequency of musculoskeletal score (31 vs. $9 \% p=0.001$ ) was higher in the former group. The frequencies of other cumulative laboratory tests and autoantibodies were similar in both groups $(p>0.0038$, Table 3).

Frequencies of cumulative treatment in cSLE patients are presented in Table 4. The frequency of use of hydroxychloroquine sulfate ( 87 vs. $59 \%, p=0.001$ ), nonsteroidal anti-inflammatory drugs (75 vs. $26 \%, p<0.0001)$ and methotrexate (47 vs. $22 \%, p=0.001$ ) were significantly higher in CA patients. 
Table 1 Chronic arthritis (CA) features in 32/852 childhood-onset systemic lupus erythematosus (cSLE)

\begin{tabular}{|c|c|}
\hline CA characteristics & $\begin{array}{l}\text { cSLE } \\
n=32(\%)\end{array}$ \\
\hline \multicolumn{2}{|l|}{ Demographic data } \\
\hline $\begin{array}{l}\text { Period between arthritis and cSLE diagnosis, } \\
\text { months }\end{array}$ & $1.0(-26$ to 93$)$ \\
\hline CA duration, months & $4.5(1.5$ to 70$)$ \\
\hline Number of joints & $6(1-26)$ \\
\hline Monoarthritis & $4(13)$ \\
\hline Oligoarthritis & $9(28)$ \\
\hline Polyarthritis & $19(59)$ \\
\hline Joints with arthritis & $236(100)$ \\
\hline Cervical spine & $2(1)$ \\
\hline Temporomandibular joint & $1(0)$ \\
\hline Shoulder & $10(4)$ \\
\hline Elbow & $13(6)$ \\
\hline Wrist & $25(11)$ \\
\hline Metacarpophalangeal joint & $43(18)$ \\
\hline Proximal interphalangeal joint & $60(25)$ \\
\hline Distal interphalangeal joint & $0(0)$ \\
\hline Hip & $10(4)$ \\
\hline Knee & $29(12)$ \\
\hline Ankle & $37(16)$ \\
\hline Tarsal joint & $6(3)$ \\
\hline \multicolumn{2}{|l|}{ Number of joints with limitation of range of motion } \\
\hline Joints with limitation of range of motion & $1.5(1-26)$ \\
\hline Cervical spine & $6(4)$ \\
\hline Temporomandibular joint & $1(1)$ \\
\hline Shoulder & $8(5)$ \\
\hline Elbow & $15(10)$ \\
\hline Wrist & $19(13)$ \\
\hline Metacarpophalangeal joint & $28(19)$ \\
\hline Proximal interphalangeal joint & $31(21)$ \\
\hline Distal interphalangeal joint & $0(0)$ \\
\hline Hip & $11(8)$ \\
\hline Knee & $7(5)$ \\
\hline Ankle & $15(10)$ \\
\hline Tarsal joint & $6(4)$ \\
\hline Deformity & $11 / 32(34)$ \\
\hline Radiographic changes* & $12 / 32(38)$ \\
\hline Presence of rheumatoid factor & $4 / 32(13)$ \\
\hline \multicolumn{2}{|l|}{ Lupus arthropathy } \\
\hline Rhupus syndrome & $6 / 32(19)$ \\
\hline Jaccoud's arthropathy & $2 / 32(6)$ \\
\hline JIA before cSLE diagnosis & $18 / 32(56)$ \\
\hline
\end{tabular}

Results are presented as median (range) and $n(\%)$

JIA juvenile idiopathic arthritis

* Juxta-articular osteopenia, joint-space narrowing and/or erosion

\section{Discussion}

Our multicenter study characterized CA as a rare manifestation of cSLE patients with a cumulative musculoskeletal damage. Patients had a predominance of a symmetric polyarthritis, involving mainly hands and ankles, and JIA was the first diagnosis in more than half of cSLE patients.

The great advantage of this study was the inclusion of a large multicentric population allowing a significant representation of $\mathrm{CA}$ in children and adolescents with cSLE, evaluating their characteristics and other possible associations. The use of a standardized database minimized any bias regarding clinical parameters and disease activity/damage score definitions. In addition, few previous reports had a clear definition of CA and rhupus in children, precluding an accurate comparison with their findings $[8,11]$. The main limitations of this study were the retrospective design and possible missing data, and the use of only one radiological imaging method. All of the participant centers are tertiary Pediatric Rheumatology referral services. These are the only centers that provide treatment for rheumatic diseases in the state of São Paulo.

CA can mislead and delay the diagnosis of lupus since JIA was the first diagnosis in more than half of these patients. We hypothesize that chronic arthritis was the first cSLE manifestation and misdiagnosed as JIA. In fact, the 1982 revised ACR criteria [9] definition states that a given criteria should be present, serially or simultaneously, during any interval of observation.

Rhupus was present in $19 \%$ of CA cases. Some studies suggest that this manifestation may distinguish a subset of patients with overlap of JIA/rheumatoid arthritis and SLE. However, there are no established definitions on whether it is an overlap condition or rhupus is in fact a spectrum of lupus musculoskeletal involvement [3-8, 11, 23]. Of note, we provide the first description of Jaccoud's arthropathy in cSLE patients characterized by a nonerosive periarticular and capsular disorder occurring as a late manifestation of disease. This condition was a very rare manifestation of cSLE with a frequency comparable to the reported in adult SLE [4, 24-27].

Importantly, SLICC/ACR-DI describes accrual damage and our cSLE patients had a significant musculoskeletal cumulative score, indicating that these patients may not have a favorable outcome due to permanent joint deformity.

In our study, the autoantibody profile did not distinguish cSLE patients with CA. In contrast, lupus arthropathy in adults was reported to be associated with specific autoantibodies profile, particularly anti-dsDNA, anti-Sm, anti-SSA/ 
Table 2 Demographic data and cumulative clinical manifestations at last visit in 852 childhood-onset systemic lupus erythematosus (cSLE) patients grouped according to chronic arthritis (CA)

\begin{tabular}{|c|c|c|c|}
\hline Variables & With CA $(n=32)$ & Without CA $(n=820)$ & $P$ \\
\hline \multicolumn{4}{|l|}{ Demographic data } \\
\hline Age at cSLE onset, years, $n=846$ & $11.5(4-17.7)$ & $11.9(0.25-17.8)$ & 0.649 \\
\hline Disease duration, years, $n=846$ & $0.2(0-15)$ & $4.6(0-23.4)$ & 0.322 \\
\hline Age at last visit, years, $n=848$ & $17.8(5-25)$ & $16.8(2-25.9)$ & 0.212 \\
\hline Time of follow-up, years, $n=846$ & $5.7(0.7-14.8)$ & $4.5(0-23.2)$ & $<0.0001$ \\
\hline Female gender & $26(81)$ & $706(86)$ & 0.439 \\
\hline Caucasian, $n=830$ & $20 / 31(65)$ & $572 / 799(72)$ & 0.393 \\
\hline \multicolumn{4}{|l|}{ Cumulative clinical manifestations } \\
\hline \multicolumn{4}{|c|}{ Constitutional features and reticuloendothelial system involvement } \\
\hline Fever, $n=848$ & $21 / 32(66)$ & $550 / 816(67)$ & 0.833 \\
\hline Weight loss $>2 \mathrm{~kg}, n=838$ & $19 / 32(59)$ & 291/806 (3) & 0.007 \\
\hline Lymphadenopathy, $n=845$ & $9 / 32(28)$ & 215/813 (26) & 0.833 \\
\hline Hepatomegaly, $n=847$ & $12 / 32(37)$ & $183 / 815(22)$ & 0.047 \\
\hline Splenomegaly, $n=846$ & 9/32 (28) & $87 / 814(11)$ & 0.002 \\
\hline \multicolumn{4}{|l|}{ Mucocutaneous involvement } \\
\hline Rash, $n=845$ & $22 / 32(69)$ & $581 / 813(14)$ & 0.739 \\
\hline Discoid lupus, $n=851$ & $1 / 32(3)$ & $53 / 819(6)$ & 0.715 \\
\hline Photosensitivity, $n=846$ & $20 / 32(62)$ & $520 / 814(1)$ & 0.873 \\
\hline Mucosal ulceration, $n=848$ & $20 / 32(62)$ & $374 / 816(46)$ & 0.064 \\
\hline Alopecia, $n=847$ & $21 / 32(66)$ & $423 / 815(52)$ & 0.127 \\
\hline Vasculitis, $n=843$ & $10 / 32(31)$ & 280/811 (34) & 0.702 \\
\hline \multicolumn{4}{|l|}{ Musculoskeletal involvement } \\
\hline Myositis, $n=845$ & $3 / 32(9)$ & $52 / 813(6)$ & 0.458 \\
\hline \multicolumn{4}{|l|}{ Serositis } \\
\hline Pleuritis, $n=845$ & $2 / 32(6)$ & $190 / 813(23)$ & 0.029 \\
\hline Pericarditis, $n=846$ & 9/32 (28) & $206 / 814(25)$ & 0.719 \\
\hline Neuropsychiatric involvement, $n=848$ & $18 / 32(56)$ & 404/816 (49) & 0.454 \\
\hline Peripheral nervous system, $n=849$ & $1 / 32(3)$ & $35 / 817(4)$ & 1.000 \\
\hline Central nervous system, $n=847$ & $18 / 32(56)$ & $398 / 815$ (49) & 0.410 \\
\hline Nephritis, $n=847$ & $20 / 32(62)$ & $533 / 815(65)$ & 0.735 \\
\hline Hematuria, $n=845$ & $18 / 32(56)$ & $516 / 813(63)$ & 0.406 \\
\hline Urine leukocytes, $n=843$ & $16 / 32(50)$ & $461 / 811(57)$ & 0.444 \\
\hline Urine casts, $n=842$ & $9 / 32(28)$ & $254 / 810(31)$ & 0.699 \\
\hline Proteinuria $>500 \mathrm{mg} /$ day, $n=830$ & $16 / 31(52)$ & 494/799 (62) & 0.252 \\
\hline Antiphospholipid syndrome, $n=784$ & $2 / 31(6)$ & $41 / 753(5)$ & 0.685 \\
\hline Ocular involvement, $n=843$ & $3 / 32(9)$ & 24/811 (3) & 0.078 \\
\hline \multicolumn{4}{|l|}{ Other } \\
\hline Arterial hypertension, $n=845$ & $15 / 31(48)$ & $331 / 814(41)$ & 0.391 \\
\hline Acute renal failure, $n=844$ & $3 / 32(9)$ & $157 / 812(19)$ & 0.247 \\
\hline Chronic renal failure, $n=841$ & $0 / 32(0)$ & $51 / 809(6)$ & 0.252 \\
\hline
\end{tabular}

The bold numbers express the $P$ values that are statistically significant Results are presented in $n(\%)$ or median (range)

$* P$ value according to Bonferroni correction for multiple comparisons $(p<0.0038)$ 
Table 3 Disease activity, cumulative damage, laboratory tests and autoantibodies evaluation at last visit in 852 childhood-onset systemic lupus erythematosus (cSLE) patients grouped according to chronic arthritis (CA)

\begin{tabular}{|c|c|c|c|}
\hline Variables & With CA $(n=32)$ & Without CA $(n=820)$ & $P$ \\
\hline \multicolumn{4}{|l|}{ Disease activity/damage scores } \\
\hline SLEDAI-2 K at first visit, $n=780 / 852$ & $12(2-40)$ & $15(0-58)$ & 0.113 \\
\hline SLEDAI- $2 \mathrm{~K}$ at last visit, $n=754 / 852$ & $4(0-16)$ & $2(0-45)$ & 0.698 \\
\hline SLICC/ACR-DI at last visit, $n=788 / 852$ & $1(0-9)$ & $0(0-7)$ & 0.003 \\
\hline \multicolumn{4}{|l|}{ SLICC/ACR-DI involvement } \\
\hline Musculoskeletal, $n=788 / 852$ & $10 / 32(31)$ & $72 / 756(9)$ & 0.001 \\
\hline Neurologic, $n=788 / 852$ & $8 / 32(25)$ & $74 / 756(10)$ & 0.006 \\
\hline Ophthalmic, $n=788 / 852$ & $6 / 32(19)$ & $65 / 756$ & 0.049 \\
\hline Renal, $n=788 / 852$ & $1 / 32(3)$ & $61 / 756(8)$ & 0.504 \\
\hline Pulmonary, $n=788 / 852$ & $0 / 32(0)$ & $8 / 756(1)$ & 1.000 \\
\hline Cardiac, $n=788 / 852$ & $2 / 32(6)$ & $18 / 756(2)$ & 0.193 \\
\hline Gastrointestinal, $n=788 / 852$ & $0 / 32(0)$ & $1 / 756(0)$ & 1.000 \\
\hline Cutaneous, $n=788 / 852$ & $3 / 32(9)$ & $18 / 756(2)$ & 0.049 \\
\hline \multicolumn{4}{|l|}{ Acute phase proteins at last visit } \\
\hline ESR mm/ $1^{\text {st }}$ hour, $n=721$ & $28(2-120)$ & $19(1-135)$ & 0.049 \\
\hline $\mathrm{CRP} \mathrm{mg} / \mathrm{dL}, n=559$ & $1(0-41)$ & $0.7(0-404)$ & 0.728 \\
\hline \multicolumn{4}{|l|}{ Cumulative laboratory tests } \\
\hline Autoimmune hemolytic anemia, $\mathrm{n}=842$ & $13 / 32(41)$ & $196 / 810(3)$ & 0.035 \\
\hline Leukopenia $<4000 / \mathrm{mm}^{3}, n=843$ & $20 / 32(62)$ & $361 / 811(44)$ & 0.045 \\
\hline Lymphopenia $<1500 / \mathrm{mm}^{3}, n=841$ & $27 / 32(84)$ & $491 / 809(61)$ & 0.007 \\
\hline Thrombocytopenia $<100,000 / \mathrm{mm}^{3}, n=843$ & $8 / 32(25)$ & $171 / 811(21)$ & 0.595 \\
\hline Low $\mathrm{C} 3, \mathrm{C} 4$ and/or $\mathrm{CH} 50, n=820$ & $24 / 32(75)$ & $562 / 788(71)$ & 0.651 \\
\hline \multicolumn{4}{|l|}{ Cumulative autoantibodies } \\
\hline Antinuclear antibodies (ANA), $n=847$ & $32 / 32(100)$ & $812 / 815(100)$ & NA \\
\hline Anti-dsDNA, $n=833$ & $25 / 32(78)$ & $576 / 801(72)$ & 0.442 \\
\hline Anti-Sm, $n=716$ & $11 / 30(37)$ & $262 / 686(38)$ & 0.866 \\
\hline Anti-RNP, $n=682$ & $12 / 29(41)$ & $183 / 653(28)$ & 0.119 \\
\hline Anti-SSA/Ro, $n=670$ & $7 / 29(24)$ & $217 / 641(34)$ & 0.278 \\
\hline Anti-SSB/La, $n=655$ & $2 / 30(7)$ & $101 / 625(16)$ & 0.205 \\
\hline Lupus anticoagulant, $n=522$ & 2/14 (14) & $105 / 508(21)$ & 0.745 \\
\hline Anticardiolipin IgM, $n=602$ & $8 / 26(31)$ & $147 / 576(25)$ & 0.549 \\
\hline Anticardiolipin IgG, $n=601$ & $12 / 26(46)$ & $175 / 575(30)$ & 0.090 \\
\hline
\end{tabular}

The bold numbers express the $P$ values that are statistically significant

Results are presented in $n(\%)$ and median (range)

SLEDAI-2K Systemic Lupus Erythematosus Disease Activity Index 2000, SLICC/ACR-DI Systemic Lupus International Collaborating Clinics/ACR-Damage Index, ESR erythrocyte sedimentation rate, CRP C-reactive protein, $N A$ not applicable to assess by Pearson $\chi$-Square or Fisher's exact test

* $P$ value according to Bonferroni correction for multiple comparisons $(p<0.0038)$
Ro, rheumatoid factor and antiphospholipid antibodies [4, $25,27]$.

As expected, treatment of CA in cSLE included nonsteroidal anti-inflammatory drugs and antimalarials in more than three-quarter of patients. The higher frequency of methotrexate use may be related to the fact that this drug was recommended for those with persistent or refractory articular manifestation [3] and the median duration of chronic arthritis observed herein was long enough to indicate this drug. 
Table 4 Cumulative therapy in 852 childhood-onset systemic lupus erythematosus (cSLE) patients grouped according to chronic arthritis $(\mathrm{CA})$

\begin{tabular}{llll}
\hline Variables & With CA $(n=32)$ & Without CA $(n=820)$ & $P$ \\
\hline Glucocorticosteroids & & & \\
Prednisone, $n=843$ & $32 / 32(100)$ & $797 / 811(98)$ & 1.000 \\
$\quad$ Current dose, $\mathrm{mg} / \mathrm{day}, n=751$ & $5(3-30)$ & $10(1-80)$ & 0.016 \\
$\quad \mathrm{mg} / \mathrm{kg} / \mathrm{day}, n=718$ & $0.10(0.03-0.7)$ & $0.2(0.02-2.6)$ & 0.129 \\
Cumulative dose, $\mathrm{g}, n=714$ & $18.5(2.7-104)$ & $19.34(0-105)$ & 0.456 \\
Intravenous methylprednisolone, $n=850$ & $25 / 32(78)$ & $558 / 818(68)$ & 0.236 \\
Cumulative dose, $\mathrm{g}, n=474$ & $15(1-79)$ & $8.73(1-139)$ & 0.057 \\
Total glucocorticoid dose, $\mathrm{g}, n=691$ & $33.4(1-129)$ & $26.11(0.4-271)$ & 0.415 \\
Hydroxychloroquine sulfate, $n=844$ & $28 / 32(87)$ & $482 / 812(59)$ & $\mathbf{0 . 0 0 1}$ \\
Nonsteroidal anti-inflammatory drugs, $n=845$ & $24 / 32(75)$ & $208 / 813(26)$ & $<\mathbf{0 . 0 0 0 1}$ \\
Intravenous immunoglobulin, $n=843$ & $3 / 32(9)$ & $65 / 811(8)$ & 0.738 \\
Immunosuppressive agents, $n=848$ & $30 / 32(94)$ & $688 / 816(84)$ & 0.209 \\
Azathioprine, $n=844$ & $21 / 32(66)$ & $494 / 812(61)$ & 0.586 \\
Cyclosporine, $n=842$ & $8 / 32(25)$ & $77 / 810(9)$ & 0.004 \\
Methotrexate, $n=842$ & $15 / 32(47)$ & $176 / 810(22)$ & $\mathbf{0 . 0 0 1}$ \\
Mycophenolate mofetil, $n=840$ & $8 / 32(25)$ & $167 / 808(21)$ & 0.554 \\
Cyclophosphamide, $n=847$ & $17 / 32(53)$ & $337 / 815(41)$ & 0.185 \\
Rituximab, $n=845$ & $1 / 32(3)$ & $8 / 813(1)$ & 0.295 \\
Plasmapheresis, $n=845$ & $0 / 32(0)$ & $24 / 813(3)$ & 1.000 \\
\hline
\end{tabular}

The bold numbers express the $P$ values that are statistically significant

Results are presented in $n(\%)$ and median (range)

$* P$ value according to Bonferroni correction for multiple comparisons $(p<0.0038)$
In conclusion, this was the first study that characterized $\mathrm{CA}$ as a rare and early manifestation of cSLE, frequently mimicking JIA at disease onset. It is predominantly polyarticular, involving more often hands and ankles and it is associated with significant musculoskeletal accrual damage.

Acknowledgments Our gratitude to Ulysses Doria-Filho for the statistical analysis. The authors thank the following Pediatric Rheumatology Divisions and colleagues for including their patients: Pediatric Rheumatology Unit, FMUSP (Adriana Almeida de Jesus, Adriana Maluf Elias Sallum, Cristina Miuki Abe Jacob, Gabriela Blay, Gabriela Nunes Leal, Gabriella Erlacher Lube de Almeida, Heloisa Helena de Souza Marques, João Domingos Montoni da Silva, Joaquim Carlos Rodrigues, Juliana Caíres de Oliveira Achili Ferreira, Laila Pinto Coelho, Luciana dos Santos Henriques, Maria Helena Vaisbich, Nadia Emi Aikawa, Lucia Maria Arruda Campos, Victor Marques, Werther Brunow de Carvalho); Pediatric Rheumatology Unit, UNIFESP (Anandreia Simões Lopes, Aline Nicácio Alencar, Claudio Arnaldo Len, Giampaolo Faquin, Gleice Clemente, Luis Eduardo Coelho Andrade, Maria Odete Esteves Hilário, Melissa Mariti Fraga, Octavio Augusto Bedin Peracchi, Vanessa Bugni); Division of Rheumatology, FMUSP (Juliane A. Paupitz, Glauce Leão Lima); UNESP (Priscila R. Aoki, Juliana de Oliveira Sato, Silvana Paula Cardin, Taciana Albuquerque Pedrosa Fernandes); Irmandade da Santa Casa de Misericórdia de São Paulo (Andressa Guariento, Maria Carolina dos Santos, Natali Weniger Spelling Gormenzano); State University of Campinas (Maraísa Centeville, Renata Barbosa); Ribeirão Preto Medical School-University of São Paulo (Gecilmara Salviatto Pileggi, Paola Pontes Pinheiro, Virginia Paes Leme Ferriani); Hospital Infantil Darcy Vargas (Jonatas Libório, Luciana Tudech Pedro Paulo); Hospital Municipal Infantil Menino
Jesus (Simone Lotufo, Tânia Caroline Monteiro de Castro) and Pontifical Catholic University of Sorocaba (Valéria C. Ramos).

Funding This study was supported by grants from Conselho Nacional de Desenvolvimento Científico e Tecnológico (CNPq 303422/2015-7 to Clovis Artur Silva, 301805/2013-0 to Rosa Maria Rodrigues Pereira, 305068/2014-8 to Eloisa Bonfá, 301479/2015 to Claudia Saad-Magalhães and 303752/2015-7 to Maria Teresa Terreri), Federico Foundation (to Clovis Artur Silva, Rosa Maria Rodrigues Pereira and Eloisa Bonfá) and by Núcleo de Apoio à Pesquisa "Saúde da Criança e do Adolescente" of USP (NAP-CriAd) to Clovis Artur Silva.

\section{Compliance with ethical standards}

Conflict of interest Ana Paula Sakamoto declares she has no conflict of interest. Clovis Artur Silva declares he has no conflict of interest. Mariana Paes Leme Ferriani declares she has no conflict of interest. Rosa Maria Rodrigues Pereira declares she has no conflict of interest. Eloisa Bonfá declares she has no conflict of interest. Claudia SaadMagalhães declares she has no conflict of interest. Eunice Okuda declares she has no conflict of interest. Simone Appenzeller declares she has no conflict of interest. Francisco Hugo Gomes declares he has no conflict of interest. Ana Luiza Garcia Cunha declares she has no conflict of interest. Mirna Henriques Tomich Salume declares she has no conflict of interest. Daniela Petry Piotto declares she has no conflict of interest. Maria Teresa Terreri declares she has no conflict of interest.

Ethical approval All procedures performed in studies involving human participants were in accordance with the ethical standards of the institutional and/or national research committee and with the 1964 
Helsinki declaration and its later amendments or comparable ethical standards.

Informed consent In all the participant centers, the Committee for Research Ethics stated that informed consent was not necessary.

\section{References}

1. Tarr T, Dérfalvi B, Györi N et al (2007) Similarities and differences between pediatric and adult patients with systemic lupus erythematosus. Lupus 0:1-8

2. Benseler SM, Silverman ED (2005) Systemic lupus erythematosus. Pediatr Clin N Am 52:443-467

3. Cavalcante EGN, Aikawa NE, Lozano RG et al (2011) Chronic polyarthritis as the first manifestation of juvenile systemic lupus erythematosus patients. Lupus 20:960-964

4. Caznoch CJ, Esmanhotto L, Silva MB et al (2006) Pattern of joint involvement in patients with systemic lupus erythematosus and its association with rheumatoid factor and hypermobility. Rev Bras Reumatol 46:261-265

5. Ziaee V, Moradinejad MH, Bayat R (2013) Rhupus syndrome in children: a case series and literature review. Case Report Rheumatol 2013:1-4

6. Unsal E, Arli AO, Akman H (2007) Rhupus arthropathy as the presenting manifestation in juvenile SLE: a case report. Pediatr Rheumatol 5:7

7. Bazsó A, Sevcic K, Orbán I et al (2011) Overlapping juvenile idiopathic arthritis and systemic lupus erythematosus: a case report. Rheumatol Int 31:695-698

8. Gormenzano NWS, Silva CA, Aikawa NE et al (2016) Chronic arthritis in systemic lupus erythematosus: distinct features in 336 paediatric and 1830 adult patients. Clin Rheumatol 35:227-231

9. Hochberg MC (1997) Updating the American College of Rheumatology revised criteria for the classification of systemic lupus erythematosus. Arthritis Rheum 40:1725

10. Silva CA, Avcin T, Brunner HI (2012) Taxonomy for systemic lupus erythematosus with onset before adulthood. Arthritis Care Res (Hoboken) 64:1787-1793

11. Pipili C, Sfritzeri A, Cholongitas E (2009) Deforming arthropathy in SLE: review in the literature apropos of one case. Rheumatol Int 29:1219-1221

12. Jaccoud FS (1869) Sur une forme de rhumatisme chronique: lecions de clinique medicale faites a l'Hopital de la Charite. Delahaye, Paris, pp 598-616

13. Gladman DD, Ibañez D, Urowitz MB (2002) Systemic lupus erythematosus disease activity index 2000. J Rheumatol 29:288-291
14. Gomes RC, Silva MF, Kozu K et al (2016) Features of 847 childhood-onset systemic lupus erythematosus patients in three age groups at diagnosis: a Brazilian multicenter study. Arthr Care Res (Hoboken). doi:10.1002/acr.22881

15. American College of Rheumatology Ad Hoc committee on neuropsychiatric Lupus Syndromes (1999) The American College of Rheumatology nomenclature and case definitions for neuropsychiatric lupus syndromes. Arthritis Rheum 42:599-608

16. Avcin T, Cimaz R, Rozman B (2009) The Ped-APS Registry: the antiphospholipid syndrome in childhood. Lupus 18:894-899

17. National High Blood Pressure Education Program Working Group on High Blood Pressure in Children and Adolescents (2004) The fourth report on the diagnosis, evaluation, and treatment of high blood pressure in children and adolescents. Pediatrics 114:555-576

18. Chan JC, Williams DM, Roth KS (2002) Kidney failure in infants and children. Pediatr Rev 23:47-60

19. Akcan-Arikan A, Zappitelli M, Loftis LL et al (2007) Modified RIFLE criteria in critically ill children with acute kidney injury. Kidney Int 71:1028-1035

20. National Kidney Foundation (2002) K/DOQI clinical practice guidelines for chronic kidney disease: evaluation, classification, and stratification. Am J Kidney Dis 39:S1-S266

21. Brandt JT, Triplett DA, Alving B et al (1995) Criteria for the diagnosis of lupus anticoagulants: an update. On behalf of the Subcommittee on Lupus Anticoagulant/Antiphospholipid Antibody of the Scientific and Standardisation Committee of the ISTH. Thromb Haemost 74:1185-1190

22. Gladman D, Ginzler E, Goldsmith C et al (1996) The development and initial validation of the Systemic Lupus International Collaborating Clinics/American College of Rheumatology damage index for systemic lupus erythematosus. Arthritis Rheum 39:363-369

23. Tani C, Dániello DD, Delle Sedie A et al (2013) Rhupus syndrome: assessment of this prevalence and its clinical and instrumental characteristics in a prospective cohort of 103 SLE patients. Autoimmun Rev 12:537-541

24. Santiago MB (2011) Jaccoud's arthropathy. Best Pract Res Clin Rheumatol 25:715-725

25. Galvão V, Atta AM, Atta MLS et al (2009) Profile of autoantibodies in Jaccoud's arthropathy. Jt Bone Spine 76:356-360

26. Ball EMA, Bell AL (2011) Lupus arthritis-do we have a clinically useful classification? Rheumatology 51:771-779

27. Cohen MR, Steiner G, Smolen JS et al (1998) Erosive arthritis in systemic lupus erythematosus: analysis of a distinct clinical and serological subset. Br J Rheumatol 37:421-424 